\title{
Interpretations of referral appropriateness by senior health managers in five PCT areas in England: a qualitative investigation
}

\author{
N Blundell, ${ }^{1}$ A Clarke, ${ }^{2}$ N Mays ${ }^{3}$
}

\section{- See linked paper on page 187}

${ }^{1}$ Research in Practice for Adults, Devon, UK

${ }^{2}$ Health Sciences Research Institute, Warwick Medical School, University of Warwick, Coventry, UK

${ }^{3}$ Health Services Research Unit, Department of Public Health and Policy, London School of Hygiene and Tropical Medicine, London, UK

\section{Correspondence to}

Dr Aileen Clarke, Health

Sciences Research Institute,

Warwick Medical School,

University of Warwick, Coventry

CV4 7AL, UK:

aileen.clarke@warwick.ac.uk

Accepted 15 November 2008

\section{(2) UN LOCK:1}

This paper is freely available online under the BMJ Journals unlocked scheme, see http:// qshc.bmi.com/site/about/ unlocked.xhtml

\section{ABSTRACT}

Aim To explore interpretations of "appropriate" and "inappropriate" elective referral from primary to secondary surgical care among senior clinical and nonclinical managers in five purposively sampled primary care trusts (PCTs) and their main associated acute hospitals in the English National Health Service (NHS).

Methods Semi-structured face-to-face interviews were undertaken with senior managerial staff from clinical and non-clinical backgrounds. Interviews were tape-recorded, transcribed and analysed according to the Framework approach developed at the National Centre for Social Research using N6 (NUD*IST6) qualitative data analysis software.

Results Twenty-two people of 23 approached were interviewed (between three and five respondents per PCT and associated acute hospital). Three attributes relating to appropriateness of referral were identified: necessity: whether a patient with given characteristics was believed suitable for referral; destination or level: where or to whom a patient should be referred; and quality (or process): how a referral was carried out, including (eg, investigations undertaken before referral, information contained in the referral and extent of patient involvement in the referral decision. Attributes were hierarchical. "Necessity" was viewed as the most fundamental attribute, followed by "destination" and, finally, "quality". In general, but not always, all three attributes were perceived as necessary for a referral to be defined as appropriate.

Conclusions For senior clinical and non-clinical managers at the local level in the English NHS, three hierarchical attributes (necessity, appropriateness of destination and quality of referral process) contributed to the overall concept of appropriateness of referral from primary to secondary surgical care.

In the UK, National Health Service (NHS) general practitioners (GPs) act as gatekeepers for the majority of non-emergency access to specialist care. Variations in referral rates from primary to secondary care have been a long-standing cause for concern both nationally and locally. Only a fraction of the variation has been demonstrated to be caused by the characteristics of patients, GPs and general practices. ${ }^{1}$ It is, therefore, widely accepted that, for many conditions, referrals may be inappropriately high or low, causing inequity in access to specialist services and inefficient use of limited healthcare resources. ${ }^{2} 3$

Appropriateness in healthcare is traditionally defined as "the ability for an individual to benefit from healthcare." ${ }^{5}$ This definition depends on definitions of benefit (eg, health gain, improvement in organ function, reduction in harmful sequelae) and definitions of healthcare, which are contextual and vary between health systems. Economic considerations are not usually included, but these clearly affect the range of healthcare options available. Other more diffuse definitions of appropriateness also exist. ${ }^{6}$

The National Institute for Health and Clinical Excellence (NICE) for England and Wales published Referral Advice, guidance intended to help local health services develop their own locally relevant referral guidelines. ${ }^{7}$ Primary care trusts (PCTs) have an overall role at the population level in the organisation and management of primary care, and the commissioning of specialist services. At the time of this study in 2005, referral management centres (RMCs) or clinical assessment services had recently been established on the initiative of many PCTs for elective referrals in order to triage referrals, thereby allowing some patients to be managed in an "intermediate" setting rather than by a hospital specialist, implying a widespread view that some referrals from primary to secondary care were inappropriate in that they did not necessarily need access to a hospital specialist service. ${ }^{8}$ In some locations, the RMC is an optional service to which GPs can refer patients for assessment and either definitive treatment by a non-consultant specialist (eg, specialist orthopaedic physiotherapist, a GP with special interest) or onward referral to a consultant. In other locations, all GP referrals are diverted to such a scheme, understandably causing frustration among GPs. ${ }^{8}$

This study was carried out as part of a broader project to develop and implement new evidencebased referral guidelines for elective surgery. The aim of the current study was to identify the interpretations and definitions of "appropriate" and "inappropriate" referral held by senior managerial staff involved in setting local referral policy at the local level in the English NHS.

\section{METHODS}

Ethical approval for the study was obtained. Semistructured qualitative interviews were carried out with senior staff in five PCTs and their associated acute hospitals between November 2004 and November 2005. Interviews were face-to-face and lasted approximately 1 hour.

\section{Topic guide}

An interview topic guide was developed using the previous literature on referral appropriateness and 
piloted. This paper focusses on participants' responses to two questions about referral appropriateness:

1. Is either of the following a cause for concern in this v:

a. Referral rates?

b. Referral appropriateness?

2. How would you define an appropriate (or inappropriate) referral?

\section{Sampling and recruitment strategy}

A purposive sampling strategy was used to select PCTs first and then senior managers within those PCTs. Five PCTs were selected on the basis of:

- Geographical distribution: a PCTwas chosen from each of the following regions: South East England, South West England, Northern England, Inner London and Outer London.

- Patient population type: information about patient population types from the Office of National Statistics' (ONS) classification of health areas was used to select PCTs representing a range of "Supergroup" categories."

- Approaches to demand management: PCTs known to be implementing explicit measures to manage demand, and others less active in the area were included.

Between three and five senior managers within PCTs and their associated acute hospitals were selected to include:

- Both clinical and non-clinical senior managers;

- Those with an interest in, or responsibility for, management of elective referrals to surgical specialties.

PCT participants we asked to nominate further possible interviewees (eg, surgeons, medical directors) from their associated acute hospital trust/s. Data collection and recruitment continued in each PCT area until we had interviewed most of those principally responsible for local referral policy. Table 1 shows the PCTs selected and the participants and their characteristics.

\section{Conduct of the interviews}

Interviews lasted about $1.5 \mathrm{~h}$ and explored the participants' interpretations of appropriateness in detail using a semi-structured topic guide, which comprised open-ended questions accompanied by probes and prompts. Interviews were conducted either individually or in groups, depending on the convenience of the respondents and their individual wishes.

\section{Data analysis}

All interviews were tape-recorded and transcribed with the participants' consent. Analysis was guided by the Framework approach developed at the National Centre for Social Research. ${ }^{10}$

Working separately first and then together, two researchers (NL and $\mathrm{AC}$ ) carried out a detailed examination of a representative subsample $(\mathrm{n}=5)$ of the interview transcripts ("Familiarization") and developed a conceptual framework, consisting of main themes and subthemes, drawing on issues introduced by the topic guide (based on a priori relationships suggested by the research literature) and also those arising within the interviews. The framework was discussed with a third member of the research team (NM) and revised to clarify shared meanings and coverage of themes and subthemes. Using the conceptual framework, all data were coded using N6 (NUD*IST version 6), and the framework adjusted or added to where necessary, checking for discrepant cases. Analysis continued until no new themes or changes to the original framework emerged. Once coded, data were transferred into structured thematic charts, which allowed easy access to condensed aggregated data relating to the themes and subthemes of interest while still retaining the participants' own language. Finally, areas where there was either clear consensus or debate were highlighted, and relationships arising out of the data (within the conceptual framework) were explored. Discussion between the researchers allowed for clarification, testing and revision of the findings.

Table 1 Characteristics of PCTs and interviewees within PCTs

\begin{tabular}{|c|c|c|c|c|c|c|}
\hline \multicolumn{7}{|c|}{ Participants: } \\
\hline PCT area & ONS supergroup & Location & ID* & PCT/acute trust & Background $\dagger$ & Job title $\ddagger$ \\
\hline \multirow[t]{3}{*}{1} & Cities and services & Outer London & P01 (i) & PCT & Non-clinical & PCT Director of Public Health \\
\hline & & & P02 (i) & PCT & Clinical $(p / t)$ & GP/Joint PCT Medical Director \\
\hline & & & P03 (i) & PCT/acute & Clinical $(p / t)$ & Joint PCT Medical Director/Consultant Physician \\
\hline \multirow[t]{4}{*}{2} & Prospering UK & N England & P04 (i) & PCT & Non-clinical & PCT Head of Primary Care \\
\hline & & & P05 (i) & PCT & Non-clinical & PCT Service Development Manager \\
\hline & & & P06 (i) & PCT & Clinical $(\mathrm{f} / \mathrm{t})$ & GP/Member of PCT Medicines Management Team \\
\hline & & & P07 (i) & Acute & Clinical $(\mathrm{f} / \mathrm{t})$ & Consultant general surgeon (with a special interest in referrals) \\
\hline \multirow[t]{5}{*}{3} & Coastal/ Countryside & SW England & $\mathrm{P} 08(\mathrm{~g})$ & Acute & Non-clinical & Director of Operations (Acute Hospital Trust) \\
\hline & & & P09 $(g)$ & Acute & Clinical $(\mathrm{p} / \mathrm{t})$ & Joint Medical Director (Acute Hospital Trust) and Consultant Physician \\
\hline & & & P10 (i) & PCT & Clinical $(\mathrm{p} / \mathrm{t})$ & Lead Commissioning GP/Vice Chair of PCT Professional Executive Committee \\
\hline & & & P11 (i) & PCT & Clinical $(\mathrm{f} / \mathrm{t})$ & GP (with a special interest in referrals) \\
\hline & & & P12 (i) & PCT & Clinical $(\mathrm{f} / \mathrm{t})$ & Specialist Physiotherapist (with a special interest in referrals) \\
\hline \multirow[t]{5}{*}{4} & Prospering UK & SE England & P13 (i) & PCT & Clinical (0) & Chief Executive of PCT \\
\hline & & & P14 (i) & Acute & Non-clinical & Director of Planned Care/Director of Tertiary Services (Acute Hospital Trust) \\
\hline & & & $\mathrm{P} 15(\mathrm{~g})$ & PCT & Non-clinical & PCT Director of Commissioning \\
\hline & & & P16 (g) & PCT & Clinical $(p / t)$ & РCT Service Development Nurse (with a special interest in referrals) \\
\hline & & & P17 (i) & Acute & Clinical $(f / t)$ & Consultant general and colorectal surgeon (with a special interest in referrals) \\
\hline \multirow[t]{5}{*}{5} & London Centre & Inner London & $\mathrm{P} 18(\mathrm{~g})$ & PCT & Clinical (0) & PCT Consultant in Public Health \\
\hline & & & $\mathrm{P} 19(\mathrm{~g})$ & PCT/acute & Clinical (0) & PCT Director of Service Development/ Acute Hospital Trust Medical Director \\
\hline & & & P20 (g) & PCT & Non-clinical & PCT Public Health Intelligence Lead \\
\hline & & & $\mathrm{P} 21(\mathrm{~g})$ & PCT & Non-clinical & PCT Public Health Information Analyst \\
\hline & & & P22 (i) & PCT & Non-clinical & PCT Public Health Information Analyst \\
\hline
\end{tabular}

GP, general practitioner; ONS, Office of National Statistics; PCTs, primary care trusts.

* $(g)$, group interview; (i) individual interview.

$\dagger \mathrm{p} / \mathrm{t}$, part time; $\mathrm{f} / \mathrm{t}$, fulltime; 0 , no current clinical commitment.

$\neq$ Job title as identified by participant. 


\section{RESULTS}

\section{Participant characteristics}

Twenty-two of 23 participants approached agreed to be interviewed (one chief executive declined but recommended a further PCT interviewee instead). Interviews were conducted in a combination of 14 individual and three group interviews. (One group consisted of four participants from the same PCT and two groups each of two participants (from a PCT and an acute trust).) Interviewees included a mixture of clinically and nonclinically trained managers from the same workplaces. Clinicians varied in the extent of their time devoted to clinical work itself. Characteristics of the participating PCTs and interviewees within PCT areas are summarised in table 1.

\section{Findings}

\section{Referral appropriateness}

The framework developed indicated three main attributes of appropriateness (figure 1). Several definitions of appropriate or inappropriate referrals appeared to be in use, the majority of which were cited by more than one participant. Examples of inappropriate rather than appropriate referrals were more frequent in the interviews.

The three distinct attributes of referral appropriateness identified by respondents were referral necessity, referral destination and referral quality. Referral necessity related to whether a patient with given characteristics was believed to be suitable for referral to a specialist; referral destination (or level) was associated with where or to whom the patient should be referred (in particular, participants queried whether consultant-level (ie, senior medical specialist) care was necessary under some circumstances, suggesting that in many cases, a non-consultant specialist, such as a physiotherapist, could provide the necessary care); and referral quality (or process) was about aspects of how a referral was carried out, including factors such as whether investigations had been undertaken before referral, or information exchanged, and the level of patient involvement in the referral decision. Attributes were hierarchical and sequential. "Necessity" was viewed as the most fundamental attribute, followed by "destination" and finally, "quality".

In general, but not universally, all three attributes were perceived as necessary for a referral to be defined as appropriate. Figure 1 illustrates how the three attributes of referral appropriateness are interconnected, hierarchical and sequential. The three attributes of appropriateness were afforded varying levels of importance by the different participants, but all were acknowledged across the various professional groups and the five PCTs.

\section{Referral necessity}

Referral necessity was the issue that most participants were most concerned about. In all five of the PCTs, there was a concern about ability to deal with "rising demand", so that when attempting to explain what constituted an appropriate referral, the participants were mostly trying to describe and define an inappropriate or unnecessary referral:

"that's a concern, an immediate concern... about demand in general;... we seem to have very high referral rates... we would like to see them reduced." (PCT5)

It was possible to distinguish between two types of necessary referral: clinically necessary referrals and referrals to manage uncertainty.

\section{Clinical necessity}

The clinical necessity of a referral was considered by participants to include:

- whether all possible primary care treatment options had been exhausted;

- whether the referral was timely;

- whether current NHS standards or guidelines (for or against referral) applied; and

- whether surgery was a likely outcome of referral.

Most participants considered a referral to be inappropriate if primary care management options had not been exhausted. The following quote highlights that referrals do take place under these circumstances but suggested that education of GPs could help:

"Dermatology is another area ... how many patients have had the appropriate primary care management before they, you know, get referred in?..." (PCT 2)

A related idea was that of the timeliness of the referral. Patients should be referred neither too early in the development of the condition, where it is still manageable in the primary care
Figure 1 Attributes of referral appropriateness.

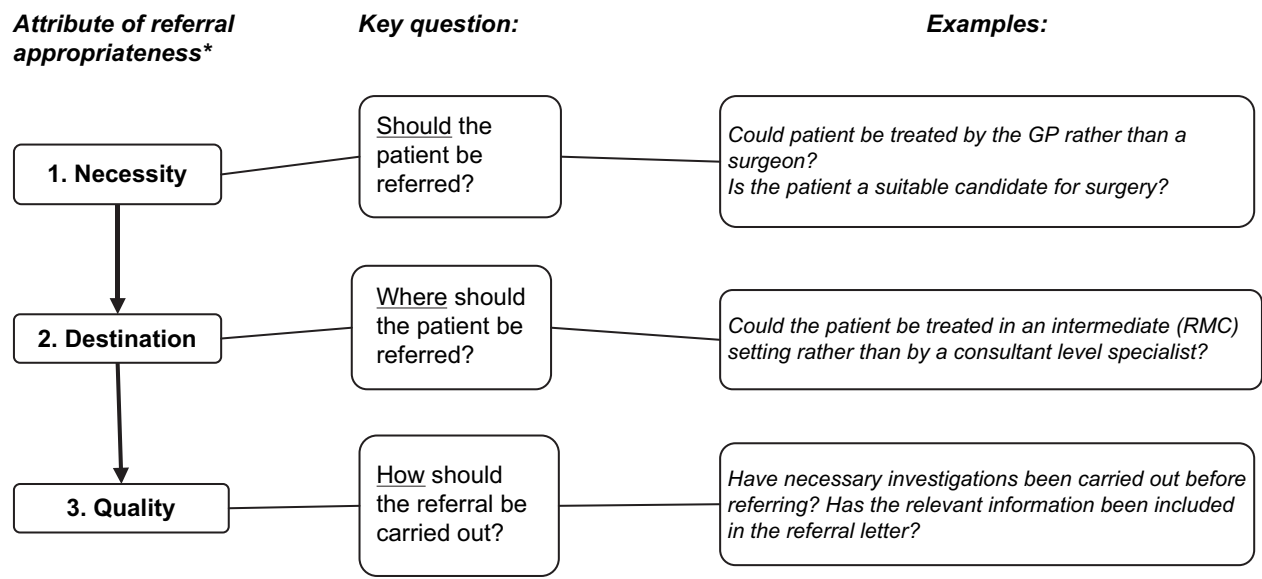

*Attributes are hierarchical: necessity needs to be present before destination can be considered; both attributes of appropriateness precede quality of process ofreferral. 
setting, nor too late, by which point they may have deteriorated too far for treatment in secondary care to be effective, as this manager explained:

\begin{abstract}
"The inappropriate [referrals]... are those people who have maybe got a chronic disease, who could have had a pathway developed... and haven't, and... get referred at a crisis point..." (PCT 3)
\end{abstract}

Some participants made reference to existing guidelines and current NHS standards, asserting that a referral was inappropriate if it did not adhere to these:

"Varicose veins is a prime example, you know, if [there's] not a clinical need... [just] a cosmetic need...." (PCT 4)

In relation to surgical referrals, a number of participants felt that GPs should pre-empt the consultant and make a judgement about whether the patient was likely to undergo surgery for their condition. They believed it was inappropriate for GPs to refer patients to surgeons if surgery was unlikely:

$$
\begin{aligned}
& \text { "... a surgeon should, as far as possible, see potential surgical } \\
& \text { patients..." (PCT 1) }
\end{aligned}
$$

But variability in individual surgeons' thresholds was also acknowledged:

\begin{abstract}
"There's about 5 or 6 different consultants,... one will operate very freely and one won't operate at all freely... sometimes it depends who that patient sees, as to what will actually happen." (PCT 3)
\end{abstract}

\section{Managing uncertainty}

A majority of participants from both clinical and managerial backgrounds acknowledged that patients are sometimes referred to manage GP or patient uncertainty and to provide reassurance. Participants felt that these referrals were, on the whole, acceptable, unavoidable and appropriate:

"...I think the thing that's often missed... referral is sometimes for a second opinion: And it isn't always for an intervention or an actual operation. It's for support of treatment." (PCT 2)

One participant highlighted the idea that a key skill for GPs is to negotiate and reassure patients, but that this is not always possible. Where the GP is certain that specialist care is not needed but the patient remains anxious and requires reassurance, this can be frustrating and can raise medico-legal concerns:

"...some people,... [demand a referral] really, if they feel so strongly then, it would be a brave GP to say "Well, I know best, you're not jolly well going"... if that's what people want,... it's very difficult to deny them." (PCT 3)

\section{Referral destination (or level)}

The second most important attribute of referral appropriateness is related to the level in the local system to which the referral should be directed. Because of the concern with large numbers of referrals considered clinically inappropriate for consultant specialist attention, many participants expressed the opinion that patients could be referred to another level: to a nonconsultant level specialist, such as a GP with a specialist interest or a community physiotherapist, who could treat or reassure the patient or provide a second opinion equally well. A range of benefits of this was asserted. Participants suggested that referral to a level other than to a senior medical specialist in secondary care might result in improved efficiency and increased convenience for the patient. “...we must do everything possible to manage... [these referrals]s. ...either in the community, or... setting up alternate services or improving the knowledge of GPs." (PCT 1)

"... the question is, does[the referral]... need to go to a consultant in an ivory tower or can you get the same reassurance from somebody with extra training and expertise in the clinical area?" (PCT3)

All five PCTs in our study had established or were developing an RMC, although each had distinct characteristics. This senior manager made explicit reference to such schemes:

"... many patients... [have] problems that can be safely managed at an intermediate tier... by someone who has... enough experience." (PCT 5)

This participant clearly believed that referrals, which in past decades would have appropriately been made to a specialist in secondary care, might now be more appropriately directed to an intermediate level, non-specialist service.

\section{Referral quality (or process)}

Lastly, participants highlighted a third attribute of appropriateness related to the process of referral. A referral could be necessary and to the right level but still be carried out in an inappropriate way. Aspects of the process of referral in this context included:

- whether necessary tests and investigations had been performed;

- whether the required information was included in the referral information;

- the extent of patient involvement in the referral decision;

- whether the referral had been sent to the correct service in secondary care;

- to whom the referral was addressed; and

- whether the referral was correctly prioritised.

The aspect of referral quality that received the most attention was the undertaking of relevant tests and investigations by GPs before making a referral.

“... some $[G P s] \ldots$ investigate their patients, and... some... don't... [although]..., some simple tests... clearly could have been done before they send [them] in." (PCT 2)

Furthermore, it was considered to be of great importance that GPs provide clear information about the patient's history and reasons for referral:

"Where there's just simply inadequate information; in other words it says: 'Dear doctor, please see and do needful, you know, yours faithfully...' That to me is an inappropriate referral, because that hasn't got enough information... " (PCT 5)

\section{DISCUSSION}

In this qualitative study of 22 senior staff with managerial responsibilities for referral policy in five PCTs in the English NHS, we aimed to understand how they conceptualised "appropriateness" (and "inappropriateness") in relation to referral from primary to secondary care for elective surgery. The definition was found to be complex and partly shaped by local context (eg, the existence of alternatives to specialist referral such as RMCs). Three sequential, hierarchical attributes of an appropriate referral were identified. These are (in order of perceived importance to our participants):

- Referral necessity-although this was of greatest concern to participants and appears to be the most important attribute, 
nevertheless, it was considered to vary between GPs and between surgeons. It consisted of two concepts: clinical necessity and managing uncertainty. It was acknowledged that some GPs have more knowledge in particular areas, and some patients require more reassurance than others.

- Referral destination or the level to which the referral is made may vary depending on the alternative services available. This definition of appropriateness depends heavily on policy change and local structures of health services. In the English NHS, the survival or demise of RMCs is likely to be influential in the future.

- Referral quality or process is obviously important and might be the easiest attribute of referral appropriateness to address, as the requirements for achieving quality of process are, on the whole, administrative and organisational. They do not require fine judgements relating to individual patients.

Participants regarded all three dimensions as relevant to appropriateness, suggesting that in assessing any referral, all three dimensions should be considered. All three require judgement to verify and are justifiably context-specific. Our participants' greatest concern in relation to appropriateness of referral appeared to be over-referral. Most gave little or no attention to under-referral or unmet need. This preoccupation with overreferral is a worrying consequence of perceptions of pressure on limited healthcare resources and emphasises the difficulty that is likely to be experienced in identifying and improving areas of under-referral and unmet need for specialist care.

There are a number of strengths to the approach used in this study. Individuals were interviewed from a geographically diverse sample of PCTs and from different senior professional backgrounds, but all involved in referral from a managerial point of view. We used a transparent, methodical, validated and widely recognised approach to analysis well-suited to health policy research. ${ }^{10}$

We intentionally interviewed those engaged at senior levels in PCTs and hospitals involved in referral policy at local level. This means that our findings do not include the views of patients, or of "ordinary" GPs or consultants. The fact that we were unable to find contrasting views on unmet need may reflect the constituency of our interviewees. Our respondents' clearly emphasised over-referral as opposed to unmet need and underreferral. There is no guarantee that ordinary GPs or specialists would have shared this preoccupation.

We were also unable to expose areas where "gaming" occurs, although there were oblique references to this. (For example, one participant talked about it in relation to the ways in which GPs might refer patients to a surgeon with a low threshold for surgery depending on their predetermined view of whether surgery was indicated or not.) It is, however, likely that some gaming occurs. ${ }^{11} 12$

We used a mixture of group and individual interviews. Group interviews afforded us (and our interviewees) efficiency of data collection and were, we believe, justified because of the lack of personal, sensitive or confidential content in the interviews. ${ }^{13}$ We found that group interviews often allowed for greater breadth of discussion, triggering recollections of particular relevant polices or issues. Substantive findings did not differ between group and individual interviews.

The qualitative approach was not designed to be statistically representative but rather to capture the likely range of views on referral appropriateness and allow for greater understanding of the concepts of appropriateness of referral held by senior staff directly involved in referral policy in a purposively chosen wide range of PCTs. The findings suggest a testable framework, which could be investigated in a quantitative survey of a larger number of respondents, both clinical and non-clinical managers, and GPs and specialists not officially involved in referral policy.

In a theoretical paper, Davies and Elwyn ${ }^{8}$ suggested three categories of inappropriate referral: those that do not conform to accepted clinical guidance, those made to the wrong service or specialty and those containing insufficient information, making it difficult to assess urgency or relevance. The findings presented in this paper support and clarify their conceptual work, giving it an empirical justification, but suggest that rather than identifying and trying to eliminate categories of inappropriate referral, it would be beneficial to regard the three positive attributes identified in this study as the test of an appropriate referral. When all three are present-the referral is necessary, directed to the correct part of the system and of good quality in process terms-there should be a greatly increased likelihood that the referral is appropriate and will result in appropriate onward healthcare.

Funding Funding was obtained from the NIHR R\&D SDO Programme. The National Co-ordinating Centre for the NIHR Service Delivery and Organisation Programme (NCCSDO), London School of Hygiene and Tropical Medicine, 99 Gower Street, London WC1E 6AA, UK, tel: 0207612 7980; fax: 0207612 7979; email: sdo@lshtm.ac.uk. We are grateful for the comments of our referees.

\section{Competing interests None.}

\section{Patient consent Obtained.}

Ethical approval was obtained from the Multi Research Ethics Committee (MREC) for Scotland.

Contributors NL was involved in study design and paper writing. She carried out data collection and led analysis. AC was the grant holder, undertook the study design, was involved in data analysis, wrote the final draft of the paper, is the corresponding author and will act as guarantor for the work. NM was involved in the study design, data analysis and paper revisions.

Provenance and peer review Not commissioned; externally peer reviewed.

\section{REFERENCES}

1. O'Donnell CA. Variation in GP referral rates: what can we learn from the literature? Fam Pract 2000;17:462-71.

2. Roland M. Measuring referral rates. In:Roland M, Coulter A, eds. Hospital referrals Oxford: Oxford University Press, 1992:62-5.

3. Wilkin D. Patterns of referral: explaining variation. In:Roland M, Coulter A, eds. Hospital referrals. Oxford: Oxford University Press, 1992.

4. Brook RH, Chassin MR, Fink A, et al. A method for the detailed assessment of the appropriateness of medical technologies. Int J Technol Assess Health Care 1986;2:53-63.

5. Lavis JN, Anderson GM. Appropriateness in health care delivery: definitions, measurement and policy implications. CMAJ 1996;154:321-8.

6. WHO. Appropriateness in health services. Report of a workshop. Koblenz (Germany): European Health 21, 2000. EUR/00/50/22388.

7. National Institute for Clinical Excellence (NICE). Referral advice: a guide to appropriate referral from general to specialist services. London: NICE, 2001.

8. Davies M, Elwyn G. Referral management centres: promising innovations or Trojan horses? BMJ 2006;332:844-6.

9. ONS. http://www.statistics.gov.uk/about/methodology by theme/ area classification/ha/cluster_summaries.asp (accessed Mar 2010). http://statistics.gov.uk/about/methodology_by_theme/area_classification/ha/ downloads/kmean8SupergroupPCT New.p̄df (accessed Mār 2010). http://www.statistics.gov.uk/about/methodology_by_theme/area_classification/ downloads/distance from centroids ha.xls (accessed Mar 2010).

10. Ritchie J, Spencer L. Qualitative data analysis for applied policy research. In:Bryman A, Burgess R, eds. Analysing qualitative data. London: Routledge, 1993:173-94.

11. Regis C. Physicians gaming the system: modern-day Robin Hood? Health Law Review 2004;13:19-24.

12. Morreim EH. Gaming the system. Dodging the rules, ruling the dodgers. Arch Intern Med 1991;151:443-7.

13. Morgan DL. Focus groups. Annu Rev Sociol 1996;22:129-52. 